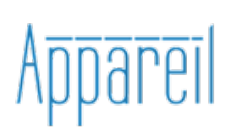

Appareil

Numéro spécial | 2008

La ville dans les sciences humaines

\title{
La ville dans les films de Guy Debord
}

\section{Véronique Fabbri}

\section{OpenEdition}

Journals

Édition électronique

URL : http://journals.openedition.org/appareil/459

DOI : 10.4000/appareil.459

ISSN : 2101-0714

Éditeur

MSH Paris Nord

Référence électronique

Véronique Fabbri, «La ville dans les films de Guy Debord », Appareil [En ligne], Numéro spécial | 2008, mis en ligne le 29 juin 2008, consulté le 30 juillet 2020. URL : http://journals.openedition.org/appareil/ 459 ; DOI : https://doi.org/10.4000/appareil.459

Ce document a été généré automatiquement le 30 juillet 2020.

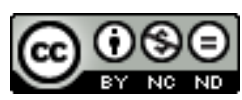

Appareil est mis à disposition selon les termes de la Licence Creative Commons Attribution - Pas d'Utilisation Commerciale - Pas de Modification 4.0 International. 


\section{La ville dans les films de Guy Debord}

\section{Véronique Fabbri}

Mon intervention lors de la journée du 25 mars consacrée à la ville, commence par la projection d'un court extrait du dernier film de Guy Debord In girum imus nocte et consumimur igni, film de 1978, dont voici deux images :

\section{Figure 1}

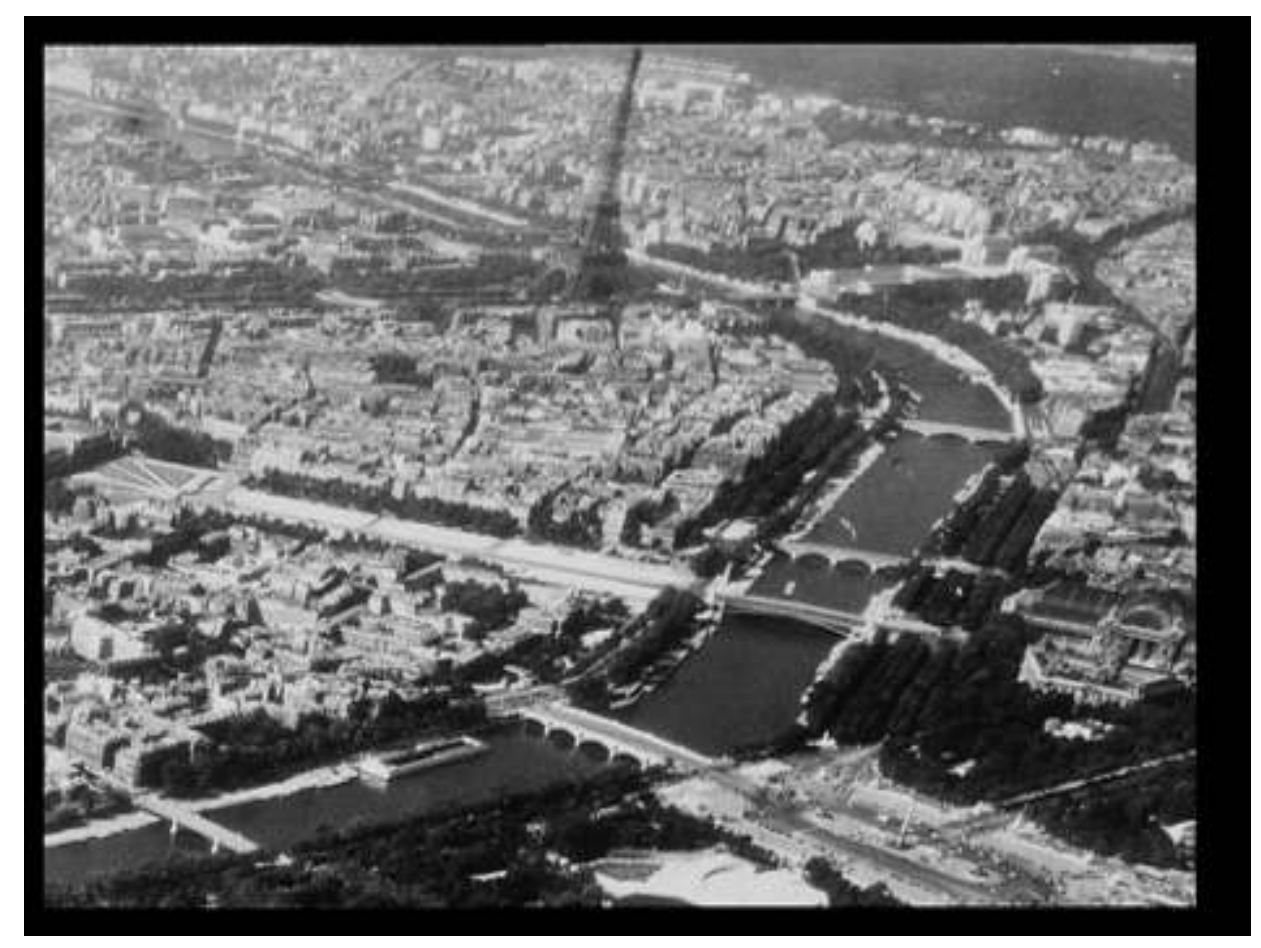


Figure 2

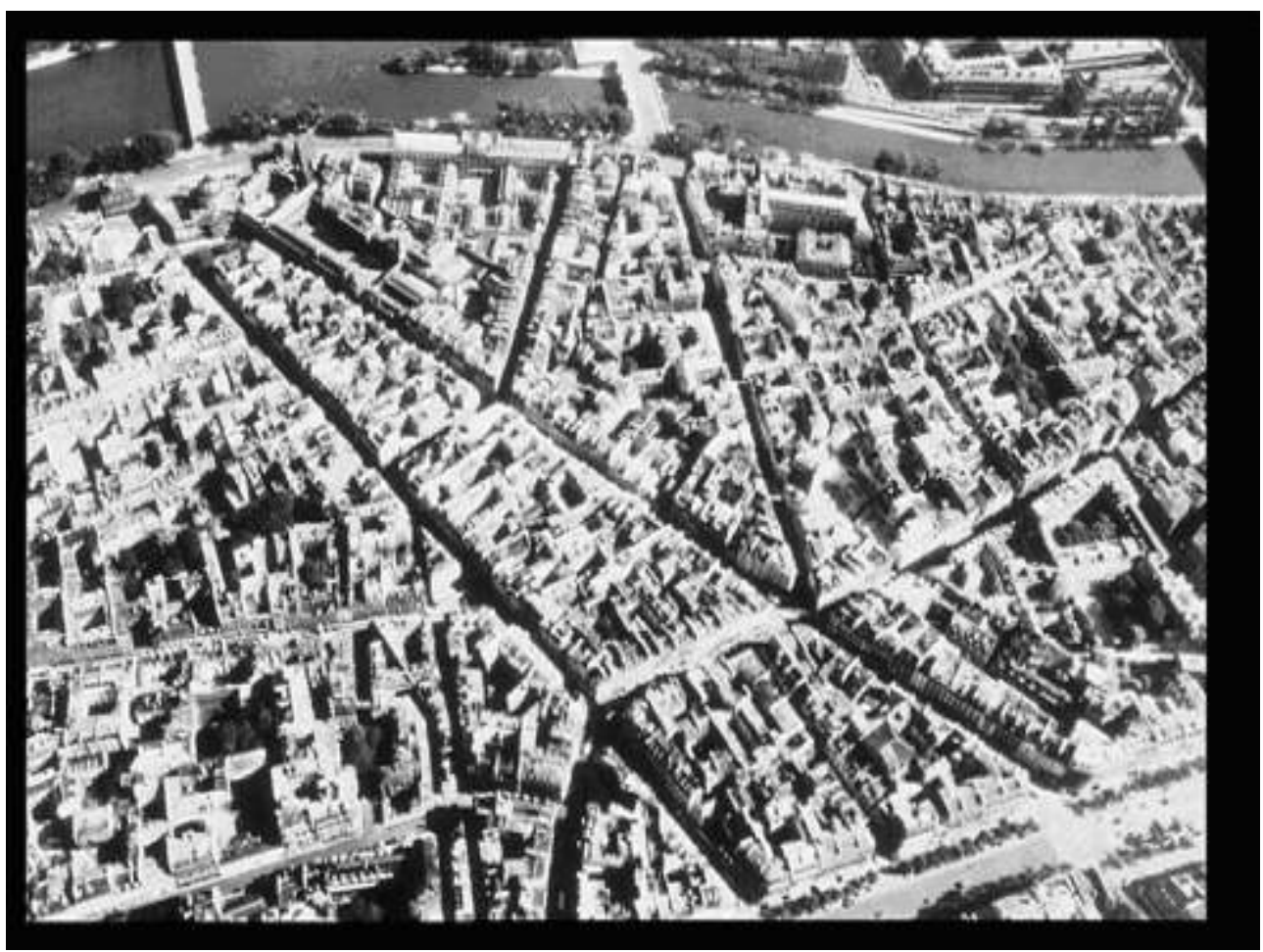

2 Le texte qui accompagne ces images est le suivant:

C'était à Paris, une ville qui était alors si belle que bien des gens ont préféré y être pauvres, plutôt que riches n'importe où ailleurs.

Qui pourrait, à présent qu'il n'en reste rien, comprendre cela; hormis ceux qui se souviennent de cette gloire? Qui d'autre pourrait savoir les fatigues et les plaisirs que nous avons connus dans ces lieux où tout est devenu si mauvais?

« ici fut la demeure antique du roi de Ou. L'herbe fleurit en paix sur ses ruines. - Là, ce profond palais des Tsin, somptueux jadis et redouté. - Tout cela est à jamais fini, tout s'écoule à la fois, les événements et les hommes, - comme ces flots incessants du Yang-Tseu-kiang, qui vont se perdre dans la mer. »

Paris alors, dans la limite de ses vingt arrondissements, ne dormait jamais tout entier, et permettait à la débauche de changer trois fois de quartier dans chaque nuit. On n'en avait pas encore chassé et dispersé les habitants. Il y restait un peuple, qui avait dix fois barricadé ses rues et mis en fuite ses rois. C'était un peuple qui ne se payait pas d'images ${ }^{1}$.

3 Cet extrait de In girum est parfois interprété comme l'expression d'une dérive de Debord vers une mélancolie de plus en plus affirmée, après ce qu'on peut considérer comme l'échec de 1968. En effet, on peut considérer que l'influence et le succès apparent des thèses situationnistes au moment de mai 68 se soldent en réalité par un échec. La société du spectacle et l'urbanisme ne cessent de se développer, Paris perd son ancienne splendeur, et la ville s'urbanise; elle entre dans l'ordre de l'urbain. L'Internationale situationniste se dissout en 1972, et les textes et productions de Debord sont de plus en plus rares. La nostalgie qu'il exprime ici à l'égard de l'ancien Paris serait en partie liée au constat d'échec des projets et propositions situationnistes. L'urbanisme semble triompher définitivement de la ville, et les marges de manœuvre pour une autre ville et une autre vie sont de plus en plus restreintes.

4 En réalité, on peut aussi bien considérer que cette nostalgie et ce ton quasi apocalyptique sont présents au début du situationnisme, et que ce film et ce texte 
révèlent peut-être d'une manière plus évidente les sources et tendances romantiques du situationnisme. Les positions du situationnisme à l'égard de la ville s'exposent ainsi à une critique plus générale que l'on peut faire des théories de Debord, critique dont on trouve des éléments chez Jean-Luc Nancy, par exemple dans Être singulier pluriel. L'envers ou l'autre du spectacle mensonger et de l'urbanisme spectaculaire serait l'expression du désir dans une imagination créatrice « dont le modèle, à tout prendre reste assez sensiblement du côté du génie romantique ${ }^{2}$ ».

5 Je souhaiterais ici examiner la portée de ces critiques et de ces questions que l'on peut adresser au situationnisme, en interrogeant les propos de Debord, en particulier dans ce film. Et ce pour la raison suivante : il me semble que malgré les critiques auxquelles s'expose de manière presque trop évidente cette pensée de la ville, est en jeu dans le situationnisme une configuration de l'art inédite, et particulièrement intéressante, un effort pour penser la relation de l'art non seulement à la vie mais à la ville, au politique. Ce qui est inédit dans cette démarche, c'est l'importance accordée ici à la forme concrète de la vie urbaine à travers les questions posées à l'urbanisme. Autrement dit, ce qui est en jeu ici, c'est à la fois une transformation de l'urbanisme par l'art et une transformation de l'art par les questions urbaines, double transformation qui ne laisse pas l'art intact dans son autonomie.

6 À cet égard, il me semble que la position de Debord est unique, et très différente, comme je le montrerai de beaucoup de démarches qui s'en inspirent, en confrontant l'artiste au milieu urbain. Il y a une manière chez Debord de penser la non-séparation de l'art et de la vie qui est très différente de l'éloge du quotidien et du banal. Et dans cette non séparation, se construit, me semble-t-il, un rapport au temps et à l'espace, qui est une pensée de l'histoire et de l'historicité, et qui ouvre des perspectives concrètes pour une action réelle dans le champ de l'urbain.

7 Je précise encore une fois que ce propos se centre principalement sur un film, et n'a pas l'ambition d'examiner l'ensemble du mouvement situationniste sur cette question.

8 Toutefois, il me faut malgré tout partir de quelques écrits antérieurs, pour comprendre la lecture et la critique que l'on peut faire des propos de Debord dans ce film, et en mesurer le sens et la portée.

\section{Debord, l'urbanisme, la critique de la société du spectacle}

9 L'extrait de film dont je pars ici, oppose, d'une manière qui peut paraitre carrément naïve, la beauté de l'ancien Paris à la laideur des nouvelles banlieues. Dès les premières années de situationnisme, on trouve une expression qui peut paraitre tout aussi schématique entre le beau et le laid, un discours quasi passéiste à l'égard de la forme que prennent les barres et grands ensembles.

10 Je cite simplement Potlatch, $\mathrm{n}^{\circ} 5$ (Revue de l'Internationale lettriste) un passage consacré à une critique de Le Corbusier.

On ne saurait oublier que si l'Urbanisme moderne n'a encore jamais été un art - et d'autant moins un cadre de vie - il a par contre été toujours inspiré par les directives de la Police ; et qu'après tout Haussmann ne nous a fait ces boulevards que pour commodément amener le canon. Mais aujourd'hui la prison devient l'habitation modèle, et la morale chrétienne triomphe sans réplique, quand on s'avise que Le Corbusier ambitionne de supprimer la rue. Car il s'en flatte. Voilà 
bien le programme: la vie définitivement partagée en îlots fermés, en société surveillées; la fin des chances d'insurrections et de rencontres; la résignation automatique ${ }^{3}$.

Si on lit de manière rhapsodique, par fragments, et dans leur diversité les textes situationnistes, on trouve qu'il y a beaucoup de lieux communs - qui ne sont pas étrangers à la pensée des théoriciens ou historiens de l'architecture et de l'urbanisme, notamment cette réduction de l'haussmanisme à une opération de police urbaine. Mais on y trouve aussi des affirmations à l'emporte-pièce qui firent fortune, notamment : cette idée que les nouveaux quartiers (zones) n'ont rien à envier à l'espace concentrationnaire et qu'on aurait pu aussi bien utiliser les "grands ensembles" comme camps de concentration.

À ces lieux communs, on peut en opposer un autre, qui relève davantage de l'esthétique classico-romantique : l'urbanisme ne pourrait se renouveler que par l'art. La critique de l'urbanisme de l'époque, se nourrit pourtant d'une réflexion sur le Bauhaus, notamment sur le premier Bauhaus, dont on retient la tentative pour former des architectes à partir du développement des pratiques artistiques, comme la peinture ou le théâtre. Ce qu'il convient de repenser, c'est la place de l'artiste dans l'urbanisme et l'architecture. Un texte d'Asger Jorn, pour un «Bauhaus imaginiste ", qualifie ainsi les artistes et leur mission : «Cette recherche doit être conduite par les artistes, avec l'aide des scientifiques", et un peu plus loin, les artistes se voient attribuer le statut d'« hommes les plus libres et les plus indépendants de la société » (1957) .

La critique de l'urbanisme sans art, dans La société du spectacle, procède d'abord de l'opposition entre la vie aliénée et la vie appropriée : l'aliénation est en partie l'œuvre de l'urbanisme moderne, si bien qu'inversement l'appropriation du milieu est la condition d'une appropriation de soi et de son histoire. La critique de l'urbanisme dans La société $d u$ spectacle, appelle d'autres pratiques urbaines par lesquelles les hommes peuvent s'approprier leur milieu, leur histoire, et ainsi se reconnaitre dans leur monde. Il s'agit de reconstruire intégralement un territoire pour y être reconnu et se reconnaître (thèse 179). De ce fait se trouve aussi reconduite l'opposition toute kantienne, entre le goût du spectacle, et le goût pur qui est réconciliation de soi avec soi.

14 De ce point de vue, le situationnisme s'expose à une critique dont on trouve des éléments décisifs chez Jean-Luc Nancy, dans Être singulier pluriel. Cette critique toutefois prend d'abord acte de la pertinence de certains éléments d'analyse proposés par le situationnisme. Elle insiste comme lui sur la misère symbolique qui mine les sociétés sans communauté. Les analyses de Jean-Luc Nancy soulignent l'insuffisance de la théorie marxiste de l'aliénation: cette dernière ne se réduit pas à une pauvreté matérielle, mais surtout à une misère symbolique :

Ce qui reste sous le pauvre mot de société, sous ce mot devenu pauvre, vidé de toute sociation, voire de toute association, pour ne rien dire des communautés et des fraternités dont on forgeait les scènes primitives... ce qui reste donc, paraît n'être rien de plus que la dite société face à elle-même, l'être-social lui-même défini par ce jeu de glaces, et se perdant dans les éclats scintillants de son miroitement. Ni l'Autre, ni les autres, mais un singulier pluriel dont l'assomption se fait par sa propre curiosité pour lui-même, dans une équivalence généralisée de toutes les représentations de soi qu'il se donne à consommer.

On a nommé cela "la société spectaculaire marchande», et «la société du $\operatorname{spectacle}^{5} »$. 

architecturaux, en particulier le fonctionnalisme. On peut en effet repérer un lien entre fonctionnalisme et marxisme.

La désappropriation pour Marx est désappropriation de l'être réel, et cet être réel est constitué de besoins concrets et d'une activité concrète. Dans l'entreprise capitaliste l'activité concrète perd son sens dans la division du travail, et dans la société marchande, les objets ne sont plus produits en vue de la satisfaction des besoins, mais d'abord en vue de la reproduction du capital. Ils deviennent des marchandises.

L'homme ne peut se réapproprier son être concret, réel, qu'en recentrant la production sur ce qui lui est nécessaire, et en réorganisant la production en fonction de sa vie réelle. Le fonctionnalisme peut être considéré comme une réponse partielle à cette exigence : il place au cœur de l'urbanisme et de l'architecture la définition des besoins vitaux, leur satisfaction et par là consonne directement avec certaines des analyses marxistes.

Mais ce processus de réappropriation de soi laisse de côté la question du symbolique ; et c'est sur ce point qu'insistent les situationnistes et Nancy. Réorganiser la production et la vie en fonction de besoins de chacun c'est en un sens manéger une place aux singularités, et sans doute, ménager la possibilité pour chacun de réinventer une forme de lien à l'autre, dans le temps libre. Mais c'est aussi ne rien dire de la nature du lien symbolique. Autrement dit, si la société marchande se symbolise dans le spectacle de la marchandise, une société fondée sur la prise en compte des besoins concrets ne se symboliserait que comme système des besoins. Cette symbolisation n'est qu'une pseudo-symbolisation : c'est la représentation d'un lien nécessaire, non la construction d'un lien symbolique.

Le problème est alors de comprendre à partir de quoi peut se constituer du symbolique : pour Jean-Luc Nancy, le symbolique est la relation même à l'autre, en tant que l'un n'existe que par l'autre; le symbolique n'est pas de l'ordre de la représentation, il est la constitution du lien. Il est l'entr'exposition des singularités. L'exposition n'est pas le spectacle, mais elle prend en compte l'idée que l'être n'est pas distinct de l'apparaître. Cette analyse du symbolique implique une critique du situationnisme au sens où la critique situationniste du spectacle supposerait toujours un être préexistant, qui est nié dans sa présentation artificielle, et la critique du spectacle serait la critique de la fausseté de cette représentation. La critique du spectacle suppose elle-même la possibilité d'une présentation vraie de soi à soi et aux autres. Les termes de vérité et de fausseté maintiennent la dissociation entre l'être et le paraître : il y aurait ainsi un paraitre adéquat et un paraître inadéquat.

À bien y regarder, on trouvera que les critiques de l'aliénation spectaculaire se fondent, et qu'elles le veuillent ou non sur la distinction d'un bon et d'un mauvais spectacle. Dans le bon spectacle, l'être social ou communautaire se présente sa propre intériorité, son origine (en soi invisible), la fondation de son droit, la vie de son corps et la splendeur de son épanouissement ${ }^{6}$.

Or, selon Nancy, l'idée d'un être originel, préexistant au spectacle est une illusion produite par le spectacle lui-même et l'insatisfaction qu'il produit. On cherche un mode d'être authentique, parce qu'on est insatisfait de l'image qu'on en a, et l'être authentique risque bien de n'être que l'image inversé du soi spectaculaire.

La dénonciation du semblant se meut elle-même dans le semblant, parce qu'elle n'a pas à désigner le propre - le non-semblant - autrement que comme l'envers obscur 
$\mathrm{du}$ spectacle. Puisque ce dernier occupe tout l'espace, son envers ne peut s'indiquer que comme l'inappropriable secret d'une propriété originaire enfouie sous les apparences ${ }^{7}$. schématique et simple. Il ne semble pas qu'il y ait chez Debord d'être, ou de sujet, qui se tienne tapis sous les apparences. S'il y a bien un bon et un mauvais spectacle, le premier est tout aussi spectacle que le second. C'est précisément un spectacle qui ne se donne que pour ce qu'il est, spectacle non d'une intériorité ou d'une vérité, mais spectacle qui n'est rien d'autre que ce qu'il est : pur jeu des apparences.

\section{Le double jeu du spectacle}

Dans In Girum, les images de Paris sont juxtaposées, confrontées à celles d'une dérive sur les canaux de Venise. Dans les deux cas, les images de ces villes sont de pures et simples apparences. Les images de Paris sont en partie hallucinatoires : la vue aérienne nous donne le sentiment d'une accumulation de ruines. La navigation sur les canaux de Venise, ne donne à voir que des façades; mais ce spectacle n'est pas un faux semblant, c'est un décor, celui d'une dérive, dont la fin sera celle du film. Venise, Debord ne peut l'ignorer, est décrite par Simmel comme la ville des façades, façades qui n'expriment ni ne dissimulent aucune intériorité :

Nous sommes captivés par la nature essentielle de cette ville, qui consiste dans une disjonction entre l'être et l'apparence, tout cela ne nous paraît exister que dans la bidimensionalité, comme quelque chose de collé sur ce qui est la nature réelle et définitive de Venise. Mais on dirait que la nature s'est consumée dans ce jeu, que chaque activité n'est qu'une façade sans arrière plan, le terme d'une comparaison dont l'autre terme aurait disparu... Les ruelles glissent comme une seule coulée. Et pareillement pour les saisons : elles glissent sur la ville, sans que le passage de l'hiver au printemps, de l'été à l'automne modifie sensiblement son image. Partout ailleurs nous sentons que la floraison et le déclin de la végétation ont une racine... Mais à Venise, c'est comme si toutes les choses avaient rassemblé toute leur beauté à leur surface, et s'étaient ensuite retirées, laissant celle-ci garder pour ainsi dire une beauté figée, soustraite qu'elle est à la vitalité et au développement de l'être réel. (p. 108, La parure et autres essais)

S'il y a un bon spectacle pour Debord, ce n'est pas celui qui rend compte d'une vérité préalable, celle de la vie réelle, mais celui qui engendre une autre vie, plus gaie et plus joyeuse: c'est celui qui révèle en acte une puissance inexplorée. Surfaces, décors, atmosphères, aspects, sont des éléments essentiels de l'architecture situationniste: celle-ci s'élabore en construisant des situations, et en expérimentant les effets qu'elles produisent.

L'animation d'une rue quelconque, l'effet psychologique de diverses surfaces et constructions, le changement rapide de l'aspect d'un espace par des éléments éphémères, la rapidité avec laquelle l'ambiance des endroits change, et les variations possibles dans l'ambiance générale de divers quartiers ${ }^{8}$.

En fait, la variété possible des combinaisons possibles d'ambiances, analogues à la dissolution des corps purs chimiques dans le nombre infini des mélanges, entraîne des sentiments aussi indifférenciés et aussi complexes que ceux que peut susciter toute autre forme de spectacle. Et la moindre prospection démystifiée fait apparaitre qu'aucune distinction qualitative ou quantitative, des influences des divers décors dans une ville ne peut se formuler à partir d'une époque ou d'un style d'architecture, encore moins à partir de conditions d'habitats9. 

populaire citadine. Mais il faut accorder la plus grande importance à la différence entre ce que dit un discours, et ce qu'il fait, surtout quand il s'écrit en contrepoint d'images. Le peuple de Paris y est évoqué selon une imagerie, ou plutôt des imageries, empruntées à la fois au cinéma et à la littérature. Ces images ne sont pas convergentes, elles forment des strates, comme une exploration archéologiques des mythes du XIX et $d u X^{e}$ siècle. Le peuple du XIX siècle est évoqué principalement à partir des images du 
film de Carné, Les enfants du Paradis. Film qui condense toute une époque de la littérature, où poètes et saltimbanques, côtoient les petits malfrats et les grands criminels, réduction du peuple à la bohême. Cette époque de la littérature est clairement analysée par Benjamin dans son travail sur Baudelaire.

Le peuple du $\mathrm{xx}^{\mathrm{e}}$ siècle en revanche est seulement évoqué dans ce texte ; il n'y est pas réellement montré. C'est d'ailleurs un peuple en voie de disparition, fantomatique. Ce qui est montré en revanche, et longuement analysé, nous y reviendrons, c'est plutôt la vie des citadins d'une cité urbanisée, classe moyenne prise dans les sortilèges de la société du spectacle. Il faut revenir au film de 59, pour avoir une idée de ce que Debord veut dire lorsqu'il parle du peuple qui alors faisait encore Paris. Le film montre la dérive d'un groupe situationniste, depuis les quartiers haussmaniens jusqu'au quartier des halles, et présente quelques beaux plans du travail matinal dans ce quartier. À cette époque donc, comme au xIX ${ }^{e}$ siècle, la survie d'un art semble liée au compagnonnage des artistes et du peuple : mais ce peuple, il faut alors le chercher pour le trouver.

Dans les deux cas, on a clairement affaire à une fiction : le peuple de Paris est fictionné comme le milieu possible d'une vie ou d'une survie de l'artiste. Il fait Paris, plus que son architecture, mais il fait Paris pour les poètes. Paris, comme le peuple de Paris, constitue un cadre de vie, en partie construit. Il y a aussi un traveling étrange qui révèle ce statut ambigu du peuple. En voici quelques images.

Figure 3

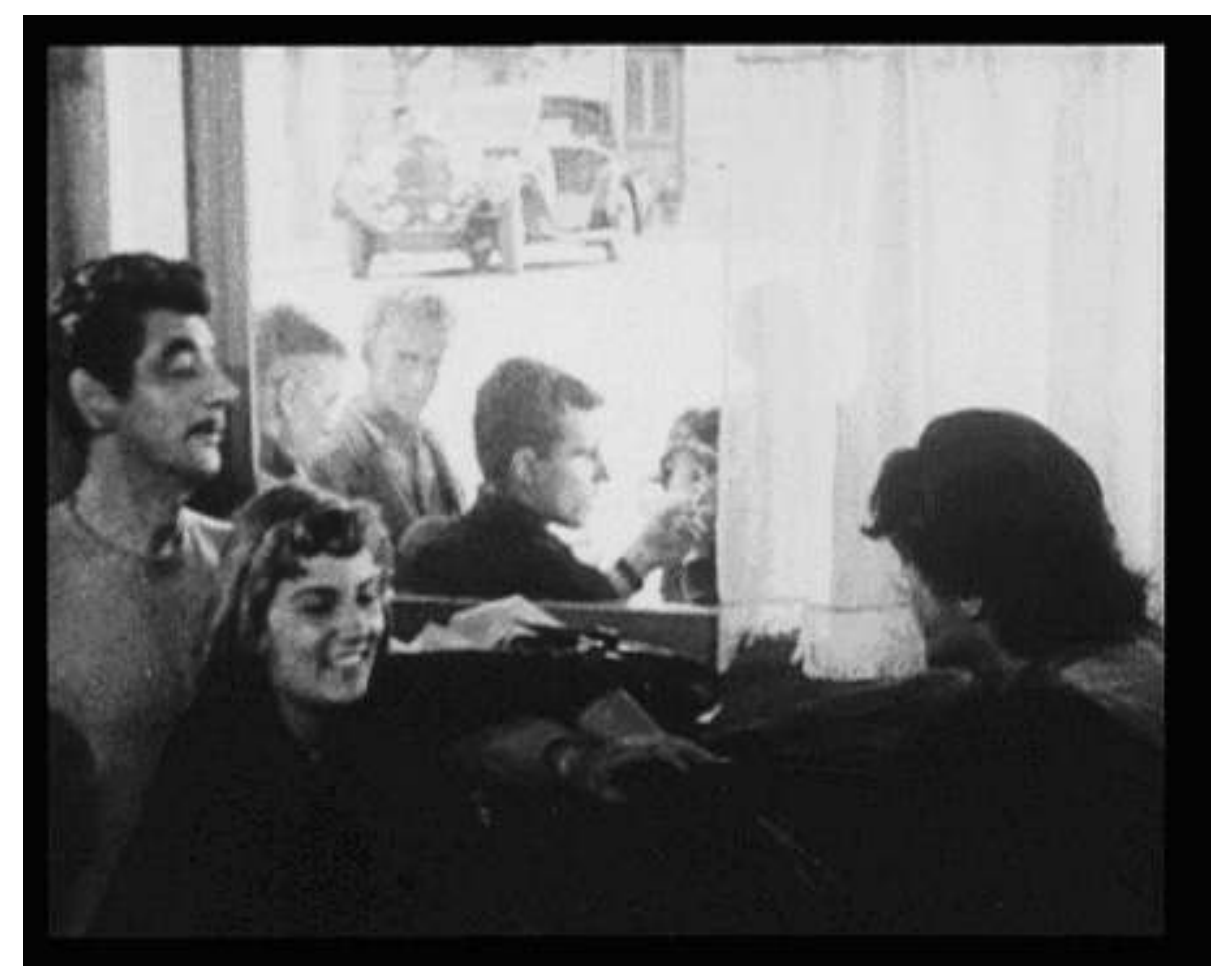


Figure 4

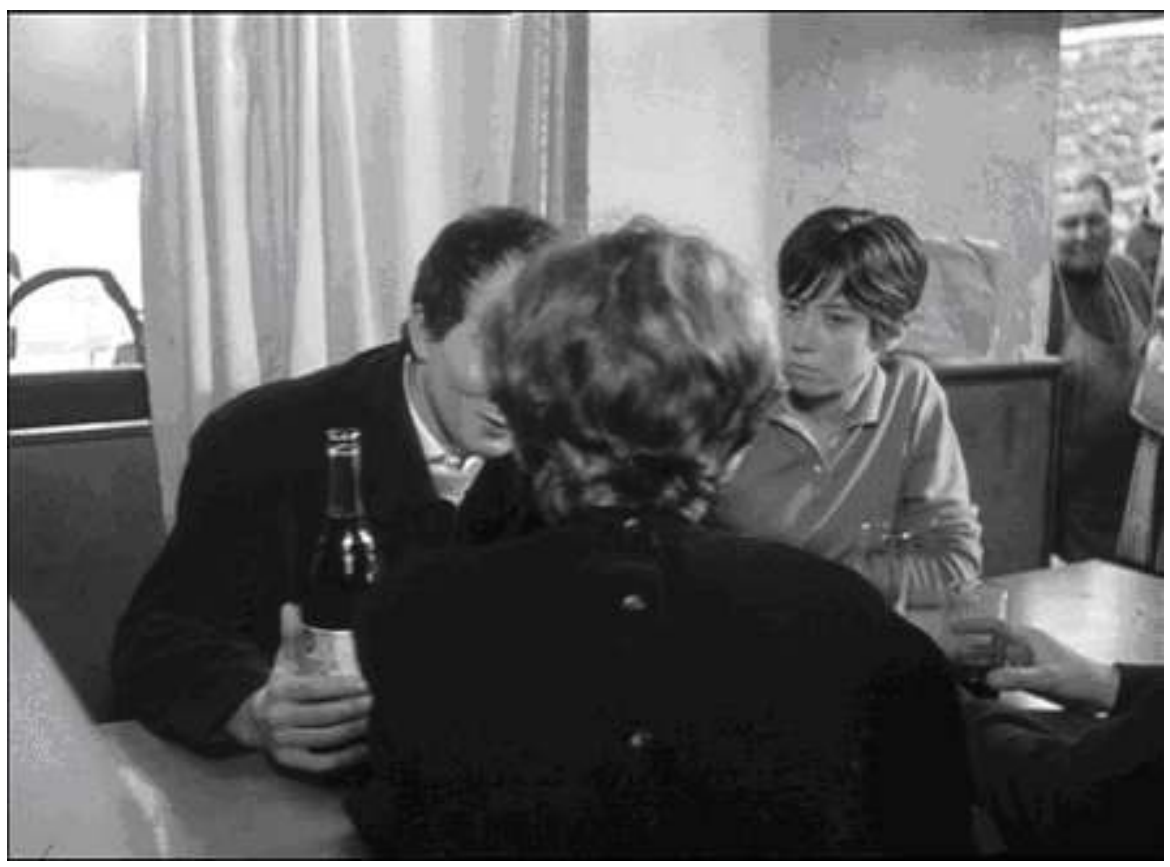
situationnistes. Ceux-ci reproduisent un comportement en phase avec une certaine idée du peuple des cafés au XIx ${ }^{e}$ siècle : « chacun buvait alors, nous dit Debord, plus de verres de vin en un jour qu'un syndicaliste ne peut dire de mensonges durant toute une grève. »

Il y va d'une fiction du peuple, qui constitue un modèle de comportement, comme l'autre du comportement bourgeois ; c'est une fiction qui est considérée comme telle, comme une construction.

Tout le début du film inversement est consacré à une analyse démystifiante des citadins, à travers une critique des images qu'on en produit: dans la critique de ces images apparaît un « quasi-peuple » qui n'a rien à voir ni avec les images publicitaires, ni avec une fiction passéiste du peuple. Ce sont des prolétaires d'une nouvelle sorte, classe moyenne parquée dans les fameux appartements concentrationnaires. Le "peuple» réel n'est plus un peuple, mais une classe indéfinissable qui ne relève d'aucune époque, entre les serfs et les péons, qui participe à son propre détriment de la société du spectacle. Pourtant c'est bien pour cette population qu'est fait le film : s'il n'y a plus de peuple, il y a bien là un public possible auquel on peut s'adresser sans concession.

Ce public contemporain, on n'en perçoit que des images, probablement empruntées à quelques magazines; c'est un public muet, dont on parle et dont on analyse les comportements, pour en montrer la misère réelle. Contrairement au peuple de Paris, le lieu où on peut le saisir est l'appartement, non la rue. Ce qui caractérise ce "quasi » peuple comme public, c'est la restriction de son espace de sociabilité à celui de la sphère intime. Mais contrairement à l'espace bourgeois de l'appartement privé, celui-ci n'est pas un intérieur qui exprimerait une intériorité. Cette sphère intime, qui est aussi son espace de sociabilité est pénétrée d'images : images diffusées par la télévision, mais aussi ensemble des objets comme marchandises. Les objets dont il s'entoure ne révèlent rien de singulier, mais un collectif qui a envahi toute la sphère privée. Entre les façades, 
les meubles, le sol carrelé, il y a reprise et répétition d'une même structure. Tout ici est façade.

L'espace urbain est fondamentalement celui du spectacle et de la façade, mais contrairement au principe du décor à Venise, ce spectacle n'est pas un espace du beau jeu des apparences. Ce spectacle est celui que l'intime se donne à lui-même, comme sa vérité, et cette vérité est celle du social ou du collectif. Ce qui caractérise le spectacle de la société spectaculaire pour Debord, c'est qu'il est spectacle de la réconciliation, de l'intérieur et de l'extérieur, du privé et du public. Son mode d'expression est le regard, et ce regard est saisi par Debord comme une extrême tension entre ce qui se dit et se montre d'une part, et ce qui n'est pas dit, d'autre part Ainsi ce jeu de regard entre une mère et son fils. Les enfants n'ont désormais que du mépris pour leurs parents péons. On n'échange plus, nous dit Debord, que des regards de haine.

En avançant dans ce film, on comprend qu'il y a sans doute pour Debord un bon et un mauvais spectacle. Mais le mauvais ne consiste pas simplement dans une falsification de ce qui est, il consiste dans le déni de la scission et de la non coïncidence à soi. Inversement, le spectacle non falsifié serait celui qui joue de cette non-coïncidence.

Les propos de Debord, qui prennent parfois l'allure d'un évangile, ne sont donc pas l'énoncé d'une vérité d'évangile. Confronté aux images toutes faites, ready-made extraits d'un flux d'images, ils deviennent plutôt des adresses et des questions. Il y a ici un double détournement, non seulement, un détournement des images et des films par le discours, mais un détournement du discours par les images. Celui-ci ne prend son sens que dans sa confrontation aux fragments de réel, dont il n'est qu'une lecture. Il est intéressant de voir que Debord a éprouvé le besoin de faire un film à partir du texte de La société du spectacle, et de mesurer la distance entre ce que dit le texte, et ce que fait le film. Le texte est pétri d'ambiguïtés que l'on a déjà soulignées avec Jean-Luc Nancy, et se prête à la répétition, la redite, comme toute sentence ou vérité sentencieuse ; le film est beaucoup plus clair et plus réjouissant. Il relève d'une stratégie du jeu. C'est dans le contrepoint des images et du texte qu'apparaît non la vérité mais un jeu sur le réel.

Mais dans les deux cas, le peuple, ou le public, occupe systématiquement la place de celui qui est privé de parole; il est, d'abord, dans les images que l'on en produit et qu'on lui renvoie de lui-même, celui qui est privé de parole.

Dans ce jeu entre une parole "sur » et des images « de », la réalité du public, du peuple de la ville et de l'urbain, perd sa consistance. Et probablement, sur ce point, l'effet du film dépasse les propos de Debord et joue contre ces propos. Le peuple et la ville se réduisent finalement à la mémoire qu'on en a et qui est fabriquée par ceux qui ont la parole ou la prennent. Il met en évidence le fait que la mémoire populaire ou comme on dit parfois aussi, la culture ouvrière n'est qu'une fiction, elle est saisie ici comme une accumulation de clichés. L'histoire inversement, est, pour Debord, une histoire qui se construit comme histoire économique, et qui est donc écrite par la bourgeoisie. On peut en conclure qu'il n'y a pas de mémoire populaire de la ville, en tout cas pas d'expérience véritable de la ville, et peut-être pas de ville du tout, au sens où tout ce que l'on en sait vient d'un regard extérieur et parfois rétrospectif : l'idée de ville n'est au fond peut-être qu'un mythe... Et peut-être ce que l'on appelle ville n'est-il que le rêve d'une ville comme condition de possibilité de l'art. Le discours sur la ville émane des artistes, cinéastes ou poètes. Le peuple ne s'approprie sa propre histoire qu'en lisant les romans, et en allant au cinéma : il y découvre qu'il a une histoire, et que la 
ville où il travaille peut être belle. Mais ces images ne correspondent pas à ce qu'il vit, et qui comme toute expérience vécue, n'est pas une expérience qui accède à la parole.

Et de même, le public qui découvre ici sa propre condition a-t-il autre chose à dire que ce qui est dit. Le mérite d'un tel film est sans doute de faire émerger l'idée d'une autre parole, un espace de jeu entre ce que l'on dit ordinairement, ce que l'on dit savamment, pour libérer l'espace de ce qui pourrait se dire autrement.

\section{La ville, espace du jeu et de la stratégie}

Il y a ainsi plusieurs idées de la ville chez Debord, et plusieurs discours de la ville. La pluralité de ces discours fait émerger une idée d'une ville comme espace de jeu : la ville n'a pas encore existé ; ce qui a existé c'est une pluralité d'idées ou d'images de la ville. C'est au moment ou émerge une autre idée, celle de l'urbain et le discours de l'urbanisme, qu'il convient de saisir la force de cette idée. Il y a ici évidemment une parenté entre cette démarche et celle de Benjamin : c'est au moment de sa disparition qu'il faut saisir ce qui est en train de disparaitre.

Dans La société du spectacle, la ville naît du développement de la production capitaliste, et elle est le lieu de formation de l'histoire historicisée. Cette histoire se construit par opposition à la temporalité cyclique du travail agricole. Sans doute toute société a-t-elle une histoire, mais certaines sociétés se construisent contre l'histoire, comme elles se construisent contre l'État. L'histoire historique suppose la perspective d'un développement irréversible et sans fin ; elle est liée à la production de plus-value, et à la gestion politique du pouvoir économique.

Le pouvoir qui s'est constitué au dessus de la pénurie du temps cyclique, la classe qui organise ce travail social et s'en approprie la plus-value limitée, s'approprie également la plus value temporelle de son organisation du temps social : elle possède pour elle seule le temps irréversible du vivant.

L'histoire universelle est née dans les villes, et elle est devenue majeure au moment de la victoire décisive de la ville sur la campagne. Marx considère comme un des plus grands mérites révolutionnaires de la bourgeoisie ce fait "qu'elle a soumis la campagne à la ville", dont l'air émancipe... La ville n'a pu être encore que le terrain de lutte de la liberté historique, et non sa possession. La ville est le milieu de l'histoire parce qu'elle est à la fois concentration du pouvoir social, qui rend possible l'entreprise historique, et conscience du passé. (thèse 176)

L'histoire historique est produite dans la ville, et le développement historique appartient essentiellement à ceux qui contrôlent le temps de travail et du développement. Dans la ville, ceux qui participent à l'histoire ne sont pas forcément ceux qui l'écrivent. Ils y participent, essentiellement comme puissance de conflit. On pourrait considérer ici que le peuple dont parle Debord, est celui qui à la fois se trouve privé de parole sur ce qui arrive, et à la fois l'auteur de ce qui arrive : il est celui par lequel l'événement est possible. Mais cet événement s'inscrit dans une histoire qui n'est pas la sienne.

Cette tension étant à terme intenable, il est possible que ceux qui font l'événement, se réapproprient l'historicité de leur vie. Dans ces conditions, l'urbanisme est analysé comme une réaction contre cette menace constituée par le rassemblement des travailleurs, qui pourraient ainsi accéder au symbolique. Il s'agit, par l'urbanisme, de produire un isolement et une séparation de ceux qui travaillent. 
L'urbanisme est l'accomplissement moderne de la tâche ininterrompue qui sauvegarde le pouvoir de classe : le maintien de l'atomisation des travailleurs que les conditions urbaines avaient dangereusement rassemblés. (172)

L'urbanisme est donc la destruction de l'urbain, la fin de l'opposition ville-campagne qui structurait l'histoire.

L'éclatement des villes sur les campagnes recouvertes de masses informes de résidus urbains est, d'une façon immédiate, présidé par les impératifs de la consommation. (174)

Cet éclatement de la ville est donc aussi la paralysie du temps historique. Il y a une relation entre une structure spatiale et urbaine et une conscience du temps, ce qui met en évidence l'articulation d'une forme du temps avec une forme de l'espace. Du coup, le temps historique apparaît comme un temps relatif, lié à une organisation du milieu géographique.

L'appropriation de l'histoire par les prolétaires doit donc passer d'abord par une appropriation de l'espace où ils vivent. Mais, paradoxalement, si on suit la logique du texte, cet espace pour une nouvelle histoire ne peut être celui de la ville, telle qu'elle s'est écrite. Si on veut être conséquent, on peut considérer que l'espace de l'urbanisme constitue le point de départ nécessaire de ce nouveau rapport à l'histoire: c'est un espace non concentré, non séparé, comme l'ancien espace défini par l'opposition villecampagne. Ce nouvel espace urbain est celui qui introduit du jeu dans l'organisation de la ville : là où il y a séparation, isolement, il $\mathrm{y}$ a aussi du jeu, ou du moins la possibilité d'un jeu. Certes, cet espace apparaît d'abord comme celui de l'uniformité, mais cette uniformité laisse dans ses marges des zones d'indétermination. Autour de ce qui est architecturé et urbanisé, il y a toujours des zones, précisément, parce qu'il n'y a pas de rues. On pourrait pour aller vite, dire qu'il s'agit d'un espace zoné : aux contours mal définis, et qui ne sont plus définis en relation à un centre. L'histoire alors relève d'une autre temporalité : elle devient plutôt l'invention hic et nunc de solutions locales.

Ce jeu trouve lui-même une forme paradoxale et contradictoire: c'est un jeu de stratégie, et c'est une stratégie qui relève de la dérive. L'espace zoné, comme le mouvement des plaques tectoniques dérive lentement, et rien n'est stable, pas même l'horizon. On n'est pas très loin de la machine de guerre selon Deleuze, à la fois art du nomadisme et art de la riposte, ou de la réponse riposte aux conditions rencontrées.

Dans In girum, la temporalité de l'histoire est constamment représentée par l'espace du jeu. Le jeu emblématique de ce film est le Kriegspiel, auquel il semble que Debord ait lui-même joué. Le propre de ce jeu, contrairement au jeu d'échecs semble être qu'il vise essentiellement à construire un espace, il vise moins la prise, que la définition imaginaire d'espaces de mouvements. L'espace de jeu est également représenté par de nombreux extraits de films comme la charge de la brigade légère. Si on met bout à bout ces séquences, ce qui apparaît, c'est la légèreté des troupes, l'isolement des combattants, et des formations qui se font et se défont.

51 Le propre d'un tel jeu, c'est qu'il n'aboutit jamais à la construction d'un espace stable, à l'instauration d'un pouvoir. Il inaugure une temporalité de la répétition, de la reprise, et du réagencement permanent.

À travers cette définition du jeu et d'une nouvelle temporalité historique, Debord nous donne probablement une des dernières définitions du rôle des avant-gardes, avant que ce terme ne tombe finalement en désuétude: "Les avant-gardes, dit-il dans ce film, n'ont qu'un temps; et ce qui peut leur arriver de plus heureux, c'est, au sens plein, 
d'avoir fait leur temps ». La temporalité selon laquelle se succèdent et se refont les avant-gardes peut définir la temporalité historique telle que l'entend Debord. Pas de pouvoir stable, mais un constant réagencement du pouvoir sur soi-même.

Dans le temps présent, dans ce qui est souvent considéré comme l'âge des masses, nous prenons volontiers l'habitude de regarder l'histoire et l'évolution comme des forces qui vont implacablement, tout à fait en dehors de notre contrôle. L'individu ressent profondément son impuissance quand il conçoit l'immensité des forces engagées. Nous les gens créatifs dans tous les domaines, devons nous défaire de cette attitude paralysante, et prendre le contrôle de l'évolution humaine en assumant le contrôle de nous-même ${ }^{10}$.

C'est une définition paradoxale d'une sorte de contre avant-garde, qui ne s'inscrit plus dans une histoire orientée, dans la perspective d'une transformation du peuple, mais d'une auto transformation partielle et incomplète de soi-même. Le paradoxe ici est aussi que la forme que prend ce contrôle de soi trouve son expression dans la pratique de la dérive.

\section{Dérive et psychogéographie}

Les moyens proposés par les situationnistes pour réformer notre rapport à l'histoire peuvent alors paraître dérisoires. La pratique de la dérive urbaine, dans les formes qu'elle prend au départ semble étonnamment naïve. Elle me paraît cependant intéressante par la place qu'elle accorde à l'urbanisme et aux transformations urbaines dans l'art. Elle inscrit la question urbaine dans l'art et non l'art dans la ville. En cela elle diffère assez radicalement de la pratique de la flânerie par les surréalistes, et de certaines tentatives des artistes, chorégraphes notamment pour investir la ville.

Avant de préciser en quoi cette théorie et pratique de la dérive diffère de la flânerie, de la marche et des performances dansées en milieu urbain, il convient de préciser en quoi elle consiste.

Debord expose cette théorie de la dérive dans le bulletin de l'Internationale situationniste, $\mathrm{n}^{\circ} 2$ de décembre 1958.

Entre les divers procédés situationnistes, la dérive se présente comme une technique du passage hâtif à travers des ambiances variées. Le concept de dérive est indissolublement lié à la reconnaissance d'effets de nature psychogéographique, et à l'affirmation d'un comportement ludique-constructif, ce qui l'oppose en tous points aux notions classiques de voyage et de promenade.

La pratique de la dérive requiert un renoncement aux motifs habituels du déplacement en ville. En revanche, elle ne consiste pas en un déplacement halluciné : elle requiert aussi une connaissance préalable du milieu auquel on se confronte. Cartes et tracés accompagnent la dérive. S'il y a un laisser-aller, il consiste dans l'expérimentation des effets psychologiques des différentes zones traversées. Mais comme expérimentation, ce laisser-aller inscrit les effets ressentis sur une carte précise. Il inscrit sur cette carte des zones d'attraction et de répulsions, une carte des passions qui se pose comme un calque à la surface de la carte initiale. La dérive est une pratique de la rencontre, mais cette rencontre convoque un hasard qui n'est pas le hasard surréaliste. Le hasard surréaliste ne note que les rencontres qui se produisent entre un inconscient singulier et certains événements ou atmosphères rencontrés dans la flânerie. Ici, dans la pratique de la dérive, il s'agit au contraire de sonder la signification anthropologique des façades et des atmosphères. La dérive relève d'une anthropomorphisation du 
monde urbain. La dérive, écrit Guy Debord, « répondrait plutôt à cette phrase de Marx : "Les hommes ne peuvent rien voir autour d'eux qui ne soit leur visage, tout leur parle d'eux-mêmes. Leur paysage est animé" ». La dérive se pratique dans un champ limité, sur un terrain balisé. En général elle part d'un point qui est une habitation, ou un lieu souvent fréquenté. Il s'agit de modifier systématiquement la perception de ce lieu habituel : l'intérêt est alors de percevoir les alentours de ce lieu, d'explorer les bords, ou le hors champ. Il n'est donc pas besoin d'un grand espace; un quartier habituel suffit. L'extension maximale est Paris et sa banlieue. Du point de vue du temps de la dérive, il n'est fixé que par l'alternance du sommeil et la veille. Si l'intensité de la dérive est suffisante, elle peut se prolonger sur plusieurs jours.

À cette théorie de la dérive, correspond une pratique de la dérive, minutieusement construite et analysée, dont un compte-rendu est publié dans le même bulletin, et rédigé par Abdelhafid Khatib. Il s'agit d'un essai de description psychogéographique des Halles, et du rôle qu'il joue dans la mythification d'un peuple. Cette dérive procède selon les normes prescrites, selon un plan précis du quartier. On commence par découper le quartier en quatre zones, selon les axes qui le traversent. Le but de ce découpage est de parvenir à une caractérisation de l'ambiance de chaque zone :

La première zone, dans l'est, est comprise entre les rues Saint-denis, de Turbigo, Pierre Lescot et la place saint Opportune. C'est la zone de la prostitution, avec une multitude de petits cafés. En fin de semaine, une foule masculine et misérable, venue d'autres quartiers, cherche à s'y divertir. Autour du square des Innocents se maintient une population de clochards. L'ensemble de cette zone es déprimant [...].

Cette première remarque tend à discréditer l'idéalisation toute mythique du peuple de paris. Au mythe, il convient de substituer une exploration réelle et éveillée de ce qui constitue l'atmosphère de Paris, et la vie populaire y apparaît d'abord comme profondément désolante. Mais cette atmosphère ne peut être analysée qu'à partir des différentes nuances qui la composent : cette atmosphère se décompose alors en zones d'intensités contrastées, dont il faut explorer la signification.

Le parcours de cette dérive à travers quatre zones repère les contrastes entre la nuit et le jour, les zones de résidence bourgeoise et les lieux de consommation, ceux du tourisme et ceux du travail. Elle repère le risque d'un déplacement progressif de la population ouvrière vers l'extérieur, mais comme on le constate, le problème n'est pas de maintenir un Paris populaire tel qu'il est. Ce Paris populaire est plutôt déprimant, jusqu'en sa pratique du divertissement ; mais l'idée du divertissement elle-même doit être maintenue. Il s'agit de conserver, contre le sérieux et l'intimité bourgeoise, un espace de divertissement collectif : c'est ce qui est retenu du peuple de Paris. Il s'agit de conserver un goût du divertissement mais de le transformer en même temps. Conserver de l'habitat populaire ce qui échappe aux contraintes du travail.

Au contraire (de l'exode) une solution qui va dans le sens d'une société nouvelle commande de conserver cet espace au centre de Paris pour les manifestations d'une vie collective libérée. Il faudrait profiter du recul de l'activité pratique alimentaire pour encourager sur une grande échelle des tendances au jeu de construction et à l'urbanisme mouvant spontanément "apparues dans les eaux glacées du calcul égoïste ». La première mesure architecturale serait évidemment le remplacement des pavillons actuels par des séries autonomes de petits édifices architecturaux situationnistes. Parmi ces architectures nouvelles et sur leur pourtour, correspondant aux quatre zones que nous avons envisagées ici, on devrait édifier des labyrinthes perpétuellement changeants à l'aide d'objets plus adéquats que les 
cageots de fruits et de légumes qui sont la matière des seules barricades

d'aujourd'hui. (p. 49)

61 intéresse les situationnistes, c'est en ce qu'elle maintient une économie qui n'est pas l'économie de laquelle elle participe. Les loisirs ne sont pas encore des loisirs rationnalisés, mais un espace et un temps où resurgit une économie archaïque. Leur analyse est en ce sens très différente de celles de l'École de Francfort, qui perçoit davantage la tristesse de ces loisirs, leur intégration progressive à une économie marchande, que leur potentiel encore subversif. Les situationnistes insistent sur le fait que le temps de récupération, même si, comme temps de récupération, il participe du fonctionnement de l'économie marchande, relève en réalité d'une autre économie qui est celle de la dépense, du don, et non de l'échange. Dans l'analyse de cette situation, on n'est pas très loin des remarques toutes chrétiennes de Hegel, concernant la peinture flamande : ce qui est peint par Jordaens, c'est bien le dimanche de la vie, jour de repos profane, consacré à la dépense et à la joie, à l'expression d'une puissance qui ne s'épuise pas toute au travail. Toutefois, le développement de l'économie signe l'arrêt d'une disparition progressive de cette économie non capitaliste. Il convient dès lors de saisir au moment où elle disparait la possibilité d'une préservation de cette économie, qui ne peut passer que par son changement et sa transformation. L'architecture et l'urbanisme sont censés constituer des remparts et des constructions pour le prolongement de ces pratiques. La pratique de la dérive est ainsi une pratique du passage du rêve et du mythe au réveil et à l'utopie. L'utopie est la formulation et la projection des désirs dans un espace construit et toute construction utopique est faite de la projection de bribes de désir. Elle comporte le sentiment de sa propre impossibilité hic et nunc. C'est pourquoi elle produit elle-même des formes quasimonstrueuses.

Ainsi, certains projets, comme ceux de Constant (New Babylon), paraissent à la fois naïfs, inconsistants, et presque monstrueux. Pourtant, ils ne le sont que parce qu'ils articulent désespérément une pratique qui disparait, à une autre qu'il s'agit de construire. En réalité, ce n'est pas le désir (ni le projet) lui-même qui est monstrueux, mais il paraît tel, parce qu'il comporte eu égard à l'espace-temps quotidien la puissance du réel, insoutenable et angoissant. Il opère une fracture dans la cohérence de l'espace vécu. Il est littéralement invivable.

On peut à partir de là préciser en quoi ces réflexions et pratiques sont résolument contemporaines, et en quoi elles se démarquent d'autres pratiques artistiques qui en paraissent très proches.

Tout d'abord, il faut noter que la pratique de la dérive s'inspire à bien des égards et plus précisément qu'on ne croit de la flânerie surréaliste. Si l'on se réfère au paysan de Paris, d'Aragon, on y trouve bien ce mélange de mythe et de rêve qui caractérise les projets situationnistes tels qu'on vient de les présenter. Contrairement à ce que l'on dit en général de la pratique surréaliste de la flânerie, celle-ci ne se limite pas à faire surgir de la ville contemporaine des mythes qui s'en étaient absentés. Elle est aussi une confrontation entre l'urbain et le non-urbain. Le chapitre consacré par Aragon au sentiment de la nature aux buttes Chaumont rejoint l'idée d'une coexistence entre une temporalité archaïque et une temporalité moderne. La différence essentielle vient surtout de ce que la pratique de la dérive est une pratique construite qui vise à situer, le mot n'est pas trop fort, les variations d'atmosphère et les effets de chaque zone. Il n'y a 
donc pas une simple coexistence d'une temporalité archaïque et d'une temporalité moderne; mais chacune d'elle renvoie à des pratiques différentes de l'économie. Cette pratique de la dérive est appareillée par un usage de la géographie, des prises de vues aériennes, mais aussi du cinéma, et enfin des textes marxistes, des considérations économiques.

La pratique de la dérive est en ce sens distincte de la flânerie. Elle rejoint les analyses de Benjamin qui montre le caractère suranné de la flânerie : l'homme moderne n'est pas le flâneur, mais celui qui se confronte aux chocs de la rue, homme qui ne reste pas à distance de la foule, suivant son propre rythme, mais s'y perd. C'est par un effort de construction et de réflexion qu'il parvient à se réapproprier une expérience qui d'abord lui échappe. Son expérience de la ville ne peut être qu'appareillée, c'est-à-dire construite après coup. Elle se fonde sur tout appareil qui met à distance l'expérience vécue, et la transforme.

Mais la principale différence entre la pratique de la dérive et les analyses de Benjamin tient à la place qui est respectivement accordée au corps dans l'une l'autre cas, et c'est là que peut s'articuler pour moi la principale critique que j'adresserai au situationnisme en général et à Debord en particulier. Chez Benjamin, la plus grande attention est apportée aux gestes, aux postures, et aux sensations kinesthésiques. Ce qu'il appelle perception distraite est une perception qui ne se focalise pas selon un seul point de vue, mais qui mobilise la mobilité même du corps, la pluralité des sensations et des formes de mémoires qui lui sont associés. Le plus étonnant inversement, chez Debord, c'est que la dérive est conçue avant tout comme une pratique psychologique, et non corporelle. L'idée même de psychogéographie indique que ce qui importe ce sont les modifications perceptives qui sont impliquées d'une zone à l'autre. Pourtant, la dérive suppose la marche. Dans le texte que j'ai cité, les seules références aux postures corporelles sont de nature biologique, elles concernent l'alternance de la veille et du sommeil. Ce que la dérive fait au corps n'est pratiquement jamais envisagé : pourtant, la marche, ou le taxi, la circulation automobile ne concernent pas que la perception, mais aussi l'inconscient corporel. De cette expérience, il n'est pratiquement jamais fait mention. Dans le film In Girum, la danse est filmée de manière ambiguë: elle est soit la danse des sauvages enfantins, soit celle des jeunes dans les boîtes, dans tous les cas elle est à la fois une figure de la dépense spontanée, et une figure du mythe, un phénomène dépourvu de conscience claire.

D'un autre côté, il me semble que Debord va plus loin dans son analyse de la dérive que bien des chorégraphes de la même époque, lorsqu'ils se confrontent au phénomène de l'urbanisation. Debord, comme De Certeau ont influencé des danseurs et chorégraphes comme Yvonne Rainer et Trisha Brown: toutes deux développent des pratiques urbaines de la danse. Mais dans les deux cas, pour ne citer que ceux-là, la ville est un terrain d'expérimentation pour une critique de la scène. La ville est en quelque sorte au service de la danse : sortir de la scène, c'est utiliser la ville comme un terrain propice à d'autres expériences du corps et notamment de la pesanteur ou des structures spatiales. Mais la danse ne produit aucune transformation de l'espace urbain. Beaucoup de pratiques actuelles sont inspirées de ce même souci. Certaines performances axées sur la marche, ont pour but de produire un état de corps ; elles ont rarement pour but de transformer et d'éclairer une perception de la ville. Par exemple, marcher sur une distance de cinq cents mètres pendant plusieurs heures, en composant la durée du mouvement de manière à faire tenir cette marche dans le temps imparti, provoque une 
concentration sur le mouvement, et annule toute perception de l'espace comme espace urbain.

Inversement, l'intérêt des analyses de Debord, et malgré la critique que je viens d'en faire, consiste à trouver une articulation de l'art au politique qui me paraît décisive. Il ne s'agit plus de déconstruire la scène, de l'ouvrir à la vie. Toutes ces questions sont toujours internes à l'art, et se perdent dans les sortilèges de la déconstruction : à force de déconstruire la scène de l'art, on en prolonge et en amplifie les principes : c'est la ville toute entière qui devient une scène pour l'art. Au contraire, l'idée de Debord est plutôt de faire de la ville une question qui s'inscrit au cœur de l'art, comme la condition de sa politisation : il s'agit d'inscrire la ville dans l'art non l'art dans la ville. Il y a donc chez Debord une politisation réussie de l'art, qui correspond à l'idée que Benjamin s'en faisait, et qui n'est en rien une esthétisation du politique. En un sens, cette politisation de l'art signifie la perte de son autonomie. Mais cette perte d'autonomie n'est pas une disparition de l'art. L'art n'est pas égal à la vie, il ne s'y confond pas: la perte d'autonomie de l'art ne signifie pas sa dissolution et son indiscernabilité.

Si l'art ne se confond pas avec la vie, ce n'est pas parce qu'elle est trop ordinaire, mais parce qu'elle lui échappe de toute part : sa puissance est plus grande que celle de l'art. Donner à voir la vie, c'est en ce sens montrer ce qui échappe au discours : dans les films de Debord, un discours se construit, savamment, et s'articule non moins savamment à une série d'images. On a trop dit que c'était un beau discours, digne de Bossuet ou de Pascal : il n'est beau que pour montrer ce qui échappe à la beauté de l'ordre. De l'un à l'autre, discours et série d'images, quelque chose manque. Ainsi, le peuple dont parle Debord n'est présent que dans le discours. Dans les images, il n'est que le fantôme de cette idée ; un peuple a disparu un autre se constitue. Le quartier de Saint-germain-després n'est présent que par quelques images qui pourraient aussi bien être tournées ailleurs. Il indique en creux une autre dérive et d'une autre ampleur. Entre les dérives locales des années cinquante, et celles qui se dessinent en 78, il y a un monde. La dérive sur le canal de Venise, dont le discours ne parle pas, cette dérive a une autre ampleur que celles qui sont construites dans le quartier des halles. C'est une dérive qui va vers les zones industrielles, ni villes, ni campagnes, à peine urbaines, se dissolvant dans le mouvement de la mer. À cette dérive répond celle qui est filmée par Antonioni dans le désert rouge. Dérive qui montre ce qui fut hors de la ville, mais devient peu à peu partie intégrante de l'urbain : ports, raffineries, et les lieux qui lui sont liés. Les images choisies par Debord sont celles qui confrontent le fragile corps humain aux forces grandissantes de la technique : il n'est plus ici question de psychogéographie, mais de la confrontation d'un corps à des forces qui le dépassent.

70 La technique utilisée par Debord n'est donc pas celle du montage, qui met en tension deux images, mais celles du détournement. Il ne s'agit pas de montrer ce qui n'est pas dit, ni de dire ce qui n'est pas montré, mais de montrer que quelque chose échappe toujours à la représentation. Ce qui importe c'est cet espace entre ce qui est dit et montré : le détournement est torsion, ou tort. Ce film ici est la figure du tort. C'est une figure de la marge, du bord, qui ne relève plus d'une imagerie de la marginalité, mais suggère, ouvre un espace pour des zones dont l'espace, la dimension, et les différences ne cessent de se creuser.

71 La seule chose dont on est sûr lorsque le film s'achève, c'est que la vie ne peut être comme elle est dite, ni comme elle est montrée ; ce qu'elle est, il nous appartient de le construire. 


\section{BIBLIOGRAPHIE}

Andreotti Libero, Le grand jeu à venir, textes situationnistes sur la ville, Paris, Éditions de La Villette, 2008.

Debord Guy, La société du spectacle, folio, 1992.

Debord Guy, CEuvres, Paris, Gallimard (Quarto), 2006.

Internationale situationniste, Paris, Arthème Fayard, 1997.

Danesi Fabien, Le mythe brisé de l'Internationale situationniste, Dijon, Les Presses du réel, 2008.

Nancy Jean-Luc, Être singulier pluriel, Paris, Galilée, 1996.

Trocchi Alexander, « Technique du coup du monde », Internationale situationniste, $\mathrm{n}^{\circ} 8$, janvier 1963.

\section{NOTES}

1. Guy Debord, CEuvres, Paris, Gallimard (Quarto), 2006, p. 1357-1358.

2. Jean-Luc Nancy, Être singulier pluriel, Paris, Galilée, 1996, p. 72-73.

3. Libero Andreotti, Le grand jeu à venir, textes situationnistes sur la ville, Paris, Éditions de La Villette, 2008, p. 73.

4. Ibid., p. 69.

5. Jean-Luc Nancy, Être singulier pluriel, p. 69.

6. Ibid., p. 90.

7. Ibid., p. 72.

8. Constant, « Le grand jeu à venir ", Potlatch, $\mathrm{n}^{\circ 0} 27$, in Libero Andreotti, Le grand jeu à venir, textes situationnistes sur la ville, Paris, Éditions de La Villette, 2008, p. 80.

9. Guy Debord, Les lèvres nues, $\mathrm{n}^{\circ} 6$, in Libero Andreotti, Le grand jeu à venir, textes situationnistes sur la ville, Paris, Éditions de La Villette, 2008, p. 82.

10. Alexander Trocchi, «Technique du coup du monde», Internationale situationniste, $\mathrm{n}^{\circ} 8$, janvier 1963.

\section{AUTEUR}

\section{VÉRONIQUE FABBRI}

Agrégée de philosophie, membre du laboratoire de philosophie de Paris 8 